\title{
A dim star with bright prospects
}

\section{A single, almost invisible star can teach us a great deal about the Solar System, other stars and the rest of our Galaxy.}

THERE are at least three reasons to be excited about the discovery, reported on page 138 of this issue by $\mathrm{B}$. Zuckerman and E.E. Becklin, of a faint companion to the white-dwarf star Giclas 29-38. First, we do not know how many dim, low-mass stars there are in the Galaxy, so we cannot say what the total mass of the Galaxy is. Second, we do not know how much mass a self-gravitating body must accumulate before its internal pressure and temperature become great enough for nuclear fusion to begin and thus a star to be born. Third, we do not know what happens to the matter that is left over as a protostar condenses out of a gaseous cloud, and whether that matter is more likely to form another star, or planets, or a ring of debris like the asteroid belt. One discovery will not answer all these questions, but observers, knowing that stars exist even though we cannot see them, will be delighted that the range of visible stars has been extended into new territory.

A brown dwarf, which is what Zuckerman and Becklin say they have found, is an object whose mass is just too small for nuclear burning to begin in earnest (Liebert, J. \& Probst, R.G. A. Rev. astr. Astrophys. 25, 473-519; 1987). It never enters the main sequence of stellar evolution, and cannot properly be called a star. It will generate some internal heat both through gravitational collapse and by the burning of deuterium, but when that meagre supply of fuel is exhausted the object must slowly cool. During its 'hot' phase, a brown dwarf can reach a temperature of $2,000-3,000 \mathrm{~K}$, and be visible as an infrared object. Zuckerman and Becklin's brown dwarf, which they saw as an infrared excess in the spectrum of an apparently normal white dwarf, seems to fit the bill fairly well. Its temperature is about $1,200 \mathrm{~K}$, its luminosity $5 \times 10^{-5}$ times the solar luminosity, and its mass $0.04-0.08$ solar masses $\left(M_{\odot}\right)$.

The uncertainty in the mass derives from uncertainties in the theoretical models with which the observations are compared. In any star, the internal temperature and density, and therefore the rate at which energy is generated, depend sensitively on the opacity of the stellar material, or in other words on the ease with which photons can escape from the core. In simple terms, the greater the opacity, the harder it is for heat to get out, making the core hotter. Opacities in normal stars can be calculated reliably from a knowledge of the abundances of the constituent elements and their ionization states; but even so, different calculations of opacity in the Sun have significant differences in the predicted rate of solar neutrino emission. For brown dwarfs, the problem is harder because they are cool enough for simple molecules to exist, and finding the opacity is a problem involving chemistry as well as physics.

It is thought that a mass of about $0.01 M_{\odot}$ distinguishes planets, which do not burn, from brown dwarfs, and that a mass of $0.08 M_{\odot}$ separates brown dwarfs from true stars. Not only are these masses uncertain, but the boundaries are in any case fuzzy. An object near $0.08 M_{\odot}$ for example, can burn hydrogen slowly for a billion years, but then expire without ever reaching a true, nuclear-burning steady state. Careful scrutiny of Zuckerman and Becklin's brown dwarf could provide one good point on the stellar-evolution graphs.

Although brown dwarfs are invisible to us unless very close, they may contribute significantly to the total mass of the Galaxy. It has been recognized since the work of Oort in the 1960 s that the dynamics of stars within a few parsecs of the Sun indicate the presence of more matter than can be accounted for by totting up all the visible stars. Very faint stars and brown dwarfs have always been popular candidates for this local 'missing' mass.

A histogram of the number of visible stars according to their mass shows that fainter stars, although individually less massive, are numerous enough to make up a larger proportion of the total galactic mass than bright stars. By continuing this trend, it is not difficult to suppose that a great deal of mass rests in brown dwarfs. On the other hand, the trend cannot continue to indefinitely small objects because they would harbour an infinite total mass. There must be a turnover in the distribution of stars at some low mass. The importance of finding this turnover has encouraged many searches for faint stars, with little success. An earlier detection by infrared speckle interferometry (McCarthy, D.W., Probst, R.G. \& Low, F.J. Astrophys. J. 290, L9-L13; 1985) of a brown-dwarf companion to the dim star Van Biesbrock 8 has, unfortunately, not stood the test of time: even its discoverers can no longer see it. Zuckerman and Becklin's discovery uses more conventional methods, and its greatest virtue may be to persuade others to keep looking.
There is no sound theoretical notion of how many low-mass objects, whether brown dwarfs or planets, we might expect to find. Because binary stars are so common, and because our own Solar System is so liberally provided with planets, moons, rings and asteroids, it is hard to escape the idea that star formation is a messy business, leaving lots of debris for other objects. But there is a more solid reason for supposing that stars are unlikely to form in isolation. As a cloud of gas begins to contract under its own gravity, any angular momentum it possesses will generate large circular velocities as the cloud shrinks, and unless the angular momentum can be disposed of, a disk, not a star, will form.

In the case of a binary star, such as Giclas 29-38, we can imagine that as the original gas cloud begins to turn into a disk, it solves its angular-momentum problem by splitting into two mutually orbiting bodies, which continue collapsing separately until they become stars. Most close binaries consist of stars of similar mass, which suggests a symmetrical formation process of this sort has occurred. On the other hand, if the total angular momentum of the collapsing cloud were smaller, it could condense further, into a flatter disk, before rotation put a stop to the process. At this point, magnetic fields could slowly transport angular momentum from the centre to the edge of the disk, allowing the central condensation to proceed towards star formation. The material in the outer disk can fragment and cool, so that planets, rather than a second star, will form. This fits the observation that the planets possess only onethousandth of the mass, but nearly all the angular momentum, of the Solar System.

In this simple scheme, every star that is not part of a binary has planets around it, and indeed the general consensus today is that planetary systems are probably common. The low-mass binary discovered by Zuckerman and Becklin may be close to the borderline between the two cases. Spectroscopy may well reveal more brown dwarfs, whereas to find planets astrometry is needed; the careful tracking of stellar positions on the sky should reveal wobbles that betray the presence of an orbiting companion. But in either case, it will be good to see old-fashioned astronomical techniques, carried out on nearby stars, answering fundamental questions about the Galaxy, and the stars and planets in it.
David Lindley 